\title{
Indikationen und essenzielle Techniken zur prähospitalen Immobilisation der Wirbelsäule
}

Anne Weißleder, Björn Hossfeld, Dominik Treffer

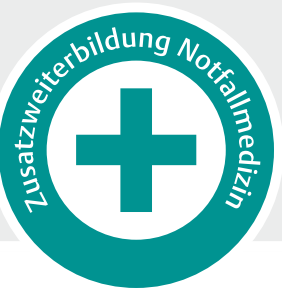

\section{Die Immobilisation der Wirbelsäule stellt eine im Rettungsdienst häufig durch- geführte Maßnahme dar. Folgender Beitrag erläutert Hintergründe und Wissens- wertes zum Wirbelsäulentrauma und beschreibt Indikationen sowie essenzielle Techniken zur Durchführung der Immobilisation in der prähospitalen Notfallmedizin.}

\begin{tabular}{|c|c|}
\hline \multicolumn{2}{|c|}{ ABKÜRZUNGEN } \\
\hline \multicolumn{2}{|c|}{$<C>A B C D E-S c h e m a$} \\
\hline & $\begin{array}{l}\text { Critical Bleeding, Airway, Breathing, Circu- } \\
\text { lation, Disability, Exposure/Enviroment }\end{array}$ \\
\hline AIS & Abbreviated Injury Scale \\
\hline BWS & Brustwirbelsäule \\
\hline CCSR & Canadian C-Spine Rule \\
\hline \multicolumn{2}{|c|}{ E. M.S. IMMO Protocol } \\
\hline & Emergency Medicine Spinal Immobilization \\
\hline & Protocol \\
\hline GCS & Glasgow Coma Scale \\
\hline HWS & Halswirbelsäule \\
\hline ICP & Intracranial Pressure (intrakranieller Druck) \\
\hline ISS & Injury Severity Score \\
\hline LWS & Lendenwirbelsäule \\
\hline MILS & manuelle In-Line-Stabilisierung \\
\hline NEXUS & $\begin{array}{l}\text { National Emergency X-Radiography Utiliza- } \\
\text { tion Study }\end{array}$ \\
\hline PHTLS $^{\circledR}$ & Prehospital Trauma Life Support \\
\hline SHT & Schädel-Hirn-Trauma \\
\hline WS & Wirbelsäule \\
\hline ZNS & Zentralnervensystem \\
\hline
\end{tabular}

\section{Einleitung}

Aufgrund der prähospital eingeschränkten Möglichkeiten müssen bei der Versorgung von Traumapatienten Verletzungsmuster antizipiert werden. So ist bei relevanter Traumakinetik ( $\triangleright$ Abb. 1) auch stets an eine Beteiligung der Wirbelsäule zu denken, zumal Verletzungen dieser in bis zu $48 \%$ der Fälle erst nach der Klinikaufnahme erkannt werden [1].

Wirbelsäulentraumata gehen gehäuft mit einer Polytraumatisierung (Injury Severity Score/ISS $\geq 16$ ) des Patienten einher. So weisen 33,6\% dieser Schwerverletzten eine ernsthafte Schädigung (Abbreviated Injury Scale/AIS $\geq 2$ ) der Wirbelsäule auf [2]. Eine begleitende Läsion des Rü- ckenmarks mit neurologischem Defizit ist bei jedem 13. dieser Patienten zu erwarten [3].

\section{Merke}

Wirbelsäulenverletzungen sind häufig Teil einer Mehrfachverletzung bzw. Polytraumatisierung und klinisch nicht bzw. nicht sicher zu erkennen.

Verkehrsunfälle und Stürze aus großer Höhe liegen dabei am häufigsten als ursächliche Traumamechanismen zugrunde. Eine Analyse schwerer Wirbelsäulentraumata (AIS $\geq 3$ ) zeigte eine annähernd gleiche Verteilung der Verletzungen auf die Hals- (34\%), Brust- $(40 \%)$ und Lendenwirbelsäule (31\%). Besonders verletzungsgefährdet sind hierbei die biomechanischen Übergänge (kraniozervikal, zervikothorakal und thorakolumbal).

\section{Merke \\ An die Möglichkeit einer begleitenden Wirbelsäulen- verletzung zu denken ist DER primär entscheidende Schritt bei der prähospitalen Versorgung von Trau- mapatienten.}

Bei vorliegendem Verdacht müssen Maßnahmen zur Ganzkörperimmobilisation durchgeführt werden. Nachfolgend werden Immobilisationstechniken der Wirbelsäule inkl. deren Vor- und Nachteile beschrieben.

\section{Ziel und Zweck}

\section{Merke}

Die Wirbelsäulenimmobilisation wird gemäß den aktuellen Leitlinien und Empfehlungen angewendet, um mögliche Sekundärschäden durch Rettung und Transport zu vermeiden, auch wenn für diese Maßnahmen keine hohe Evidenz besteht [4-6].

Maßgeblich für die Auswahl des Immobilisationsverfahrens sind:

- der Patientenzustand, 


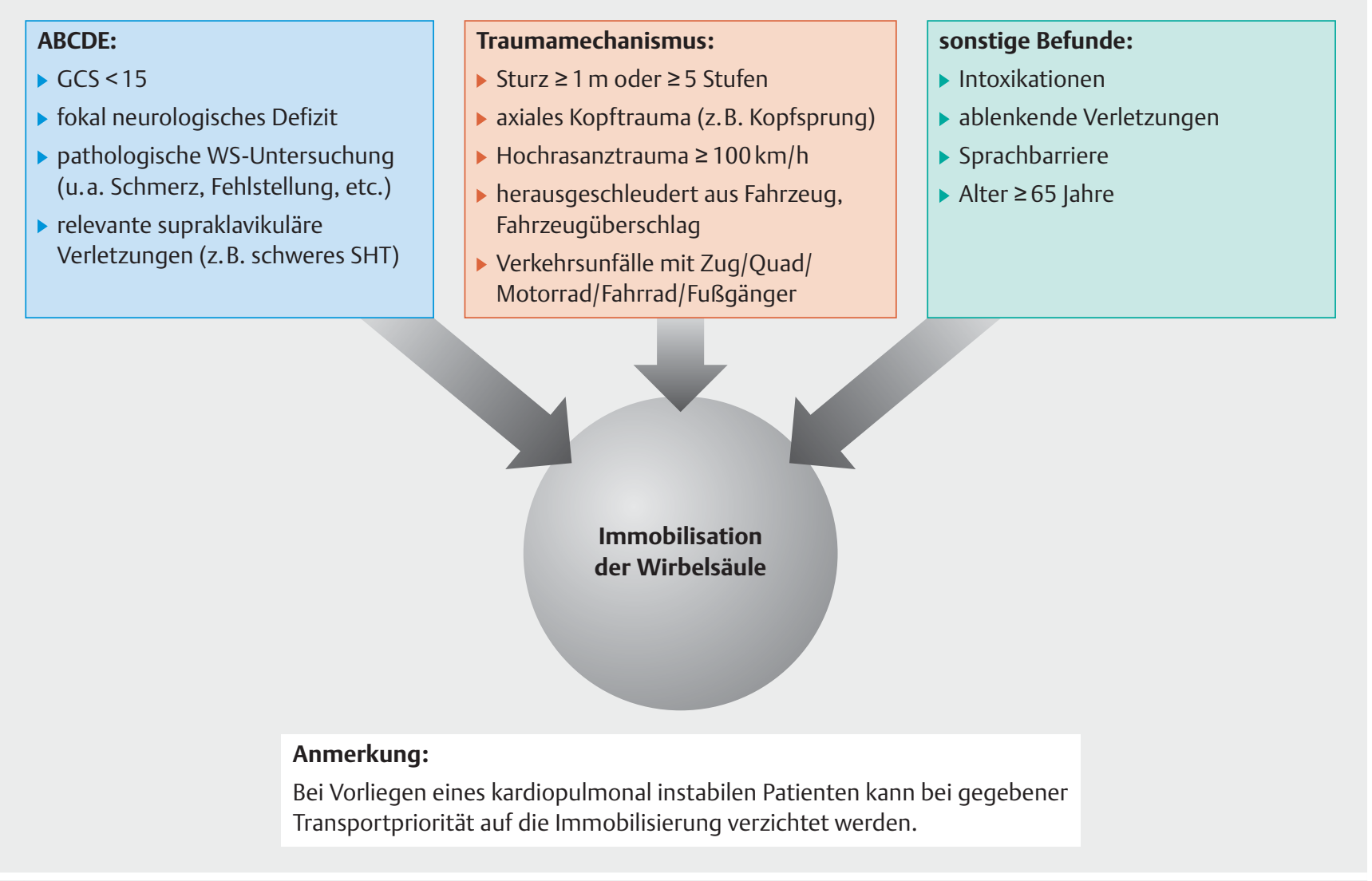

- Abb. 1 Indikationen zur prähospitalen WS-Immobilisation (nach [6, 15, 16].

- das Einsatzumfeld,

- die Erfahrung des Rettungsteams sowie

- die Ausstattung des Rettungsmittels.

Während bei akut vital bedrohten Patienten (A/B/C/DProblem) bzw. gegenwärtiger Gefahrenlage am Einsatzort (z.B. brennendes Fahrzeug) die schonende Rettung mit Schutz der Wirbelsäule in den Hintergrund tritt, so hat sie unter kontrollierten Einsatzbedingungen und bei vital stabilen Patienten höchste Priorität. Die Übersicht „Verfahren der Rettung“ beschreibt die unterschiedlichen Vorgehensweisen anhand des Patientenzustandes.

\section{ÜBERSICHT}

Verfahren der Rettung (nach [7])

- sofortige (Crash-) Rettung

- bei akuter Lebensgefahr (keine WS-Immobilisation möglich)

- schnelle Rettung

- bei potenziell kritischen Patienten (weitestmögliche Schonung der Wirbelsäule)

- schonende Rettung

- bei stabilem Patientenzustand (Fokus auf konsequente Wirbelsäulenimmobilisation)
Die sinnvolle Auswahl und Planung des Immobilisationsverfahrens sowie die Kommunikation im Team sind von allergrößter Bedeutung für den Erfolg der Maßnahme.

\section{Prähospitales Vorgehen entsprechend dem $<$ C $>$ ABCDE-Schema}

Die initiale Untersuchung und Therapie des Traumapatienten („Primary Assessment“) erfolgt nach prioritätenbasierten Versorgungsalgorithmen (z. B. <C>ABCDE gem. $\left.\mathrm{PHTLS}^{\circledR}[8]\right)$. Sobald die Sicherheit an der Einsatzstelle gewährleistet ist, muss der Unfallmechanismus und klinische Ersteindruck („general Impression“) des Traumapatienten erfasst werden. Die Sicherung der Vitalfunktionen steht im nun durchzuführenden Primary Assessment im Vordergrund [7]. Im Folgenden werden Befunde und Maßnahmen fokussiert auf Wirbelsäulenverletzungen nach $<C>A B C D E$ gegliedert vorgestellt:

\section{$<\mathrm{C}>-$ Critical Bleeding}

Stillen bedrohlicher, externer Blutungen. 


\section{A - Airway: Untersuchung und Sicherung des Atemwegs sowie HWS-Stabilisierung}

Es erfolgt die Untersuchung und ggf. Sicherung des Atemwegs sowie die Halswirbelsäulenstabilisierung. In Hinblick auf mögliche Wirbelsäulenverletzungen müssen bei der Untersuchung und Sicherung des Atemwegs Bewegungen in der Halswirbelsäule, insbesondere eine Flexion, vermieden werden. Etablierte Techniken sind die manuelle Immobilisation (Manuelle In-Line-Stabilisierung - MILS) der HWS sowie die Anlage einer Zervikalstütze [7].

\section{Cave}

Die alleinige Anlage einer Zervikalstütze ist keine ausreichende Stabilisierung der HWS und muss stets mit einer Ganzkörperimmobilisation kombiniert werden [5-7]. Zu bedenken ist ebenfalls, dass Verletzungen der Halswirbelsäule in ca. 10,8\% der Fälle mit weiteren Verletzungen der Wirbelsäule einhergehen [9].

\section{B - Breathing: Überprüfung der Atemfunktion}

Pathologische Atemmuster (z.B. Cheyne-Stokes-Atmung) treten mitunter als Folge einer Schädigung des Atemzentrums im Hirnstamm auf. Läsionen im Bereich des Zervikal- und Thorakalmarks können die Atmung durch eine Beeinträchtigung der Atemmuskulatur erschweren. Die Verletzung des Rückenmarks im Bereich von C3-C5 (Ursprung des N. phrenicus) kann in eine Lähmung des Zwerchfells mit konsekutivem Atemstillstand münden [10].

\section{C - Circulation: Evaluation der Hämodynamik}

Der neurogene Schock stellt in diesem Kontext eine relevante C-Problematik dar. Klinisch imponiert das gleichzeitige Auftreten einer Hypotonie und Bradykardie, aufgrund des Verlusts der sympathischen Innervation des Herzens (insbesondere fehlende positive Ino- und Chronotropie) sowie der Gefäßmuskulatur (Vasoplegie).

\section{D - Disability: neurologische Beurteilung}

Die Prüfung der Bewusstseinslage mit Erhebung der Glasgow Coma Scale (GCS) und die Pupillenkontrolle sind wesentliche Bestandteile der neurologischen Untersuchung. Bei Vorliegen eines sensiblen und/oder motorischen Defizits kann versucht werden, die Lokalisation der Myelonschädigung anhand von Dermatomen und Kennmuskeln zu evaluieren [10].

\section{Cave}

Bei Patienten mit relevantem Schädel-Hirn-Trauma (SHT) droht eine sekundäre Hirnschädigung durch Erhöhung des intrakraniellen Drucks (ICP). In diesen Fällen ist die Anlage einer Zervikalstütze kritisch zu überdenken (ICP-Erhöhung durch reduzierten venösen Abfluss) und ggf. eine alternative Immobilisa- tionsmethode zu wählen (z. B. Vakuummatratze oder Spineboard mit Head Blocks) [5-7].

\section{E - Exposure \& Environment: Entkleiden, Wärmeerhalt und erweiterte Untersuchung}

Zur Detektion weiterer Verletzungen muss der Patient bedarfsgerecht entkleidet werden. Wesentlich ist hierbei die Untersuchung von Rücken und Wirbelsäule. Hierfür muss eine achsengerechte Drehung des Patienten durch mehrere Helfer, unter konsequenter Stabilisierung der Halswirbelsäule (MILS) erfolgen. Die Inspektion (z. B. Hämatome, Fehlstellungen) und Palpation (z.B. Druck-/ Klopfschmerz, Instabilität) können auf Verletzungen der Wirbelsäule hinweisen. Studien zeigen jedoch eine geringe Sensitivität für die klinische Untersuchung der thorakolumbalen Wirbelsäule, insbesondere bei bewusstlosen Traumapatienten $[12,13]$. Zugleich erfolgt die Inspektion der Perianalregion (Stuhl-/Urinabgang, Priapismus etc.). Die digital-rektale Untersuchung hat hierbei eine geringe Sensitivität in der Detektion traumatischer Rückenmarkverletzungen [14].

\section{Indikationen und Kontraindikationen der Wirbelsäulenimmobilisation}

Wie jede medizinische Maßnahme bedarf auch die Ganzkörperimmobilisation einer rechtfertigenden Indikation, da sie mit weitreichenden Folgen für das weitere präwie innerhospitale Management verbunden ist (z.B. erschwerte Atemwegssicherung, prolongierte Versorgungsdauer) [4-6].

Die Canadian C-Spine-Rule (CCSR) und die Kriterien gemäß National Emergency X-Radiography Utilization Study (NEXUS) wurden ursprünglich als klinische Entscheidungshilfen zur Notwendigkeit einer radiologischen Diagnostik der Halswirbelsäule nach Trauma entwickelt $[15,16]$. Eine Validierung als prähospitale Maßgabe zur Wirbelsäulenimmobilisation erfolgte lediglich für die CCSR [16]. Das neu entwickelte Emergency Medicine Spinal Immobilization Protocol (E. M. S. IMMO Protocol) steht seit 2016 als weiterer Algorithmus zur Verfügung [6]. > Abb. 1 fasst die wesentlichen Indikationen in Anlehnung an die o.g. Protokolle zusammen.

\footnotetext{
Cave

Eine prolongierte Liegedauer auf dem Spineboard provoziert Hypoperfusion und Schmerzen im Bereich der aufliegenden Körperstellen. Die Vakuummatratze bietet hier mehr Komfort und einen höheren Grad der Immobilisierung $[5,6,17]$.
} 


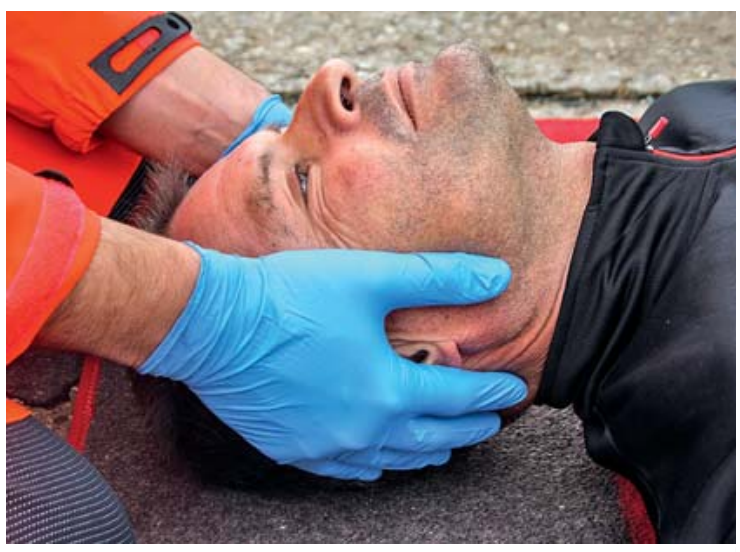

- Abb. 2 Manuelle In-Line-Stabilisierung (MILS) der Halswirbelsäule.

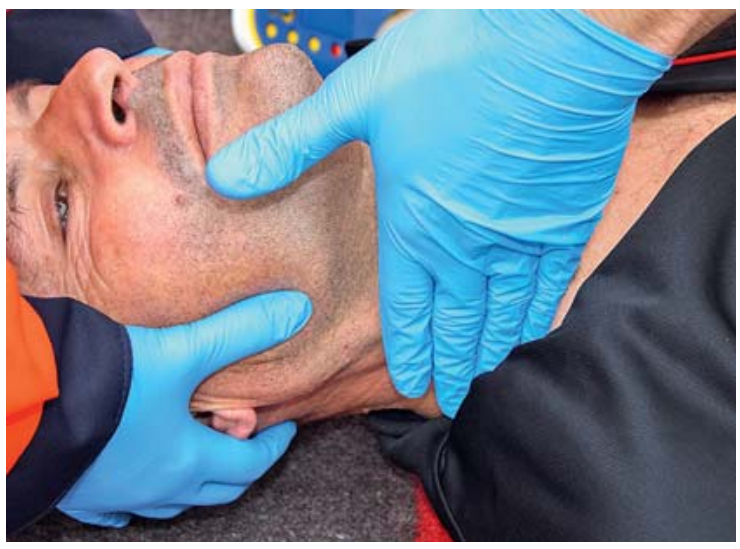

- Abb. 3 Abmessen der Distanz Schulteransatz-Kinn am Patienten in Normalposition des Kopfes mithilfe der Finger des Helfers unter MILS.

\section{Techniken}

Nachfolgend werden einige essenzielle Techniken zur Rettung und zum Transport des Patienten mit vermutetem Wirbelsäulentrauma dargestellt.

\section{Stabilisierung der Halswirbelsäule}

Schritt 1 Manuelle In-Line-Stabilisierung

Als erstes wird die HWS behutsam in Neutralposition verbracht, wenn dies schmerzfrei und ohne Zunahme eines neurologischen Defizits möglich ist. $>$ Abb. 2 zeigt die manuelle In-Line-Stabilisierung (MILS) der Halswirbelsäule in Neutralposition durch einen Helfer.

\section{Schritt 2 Ausmessen der Zervikalstütze}

Nun können die Distanz zwischen Schulteransatz und Kinn des Patienten bestimmt ( $\triangleright$ Abb. 3 ) und die Größe der Zervikalstütze eingestellt werden ( $\bullet$ Abb.4); ggf. sind weitere Vorbereitung nach Herstellerangaben zu treffen, z. B. eine Verriegelung der Halskrause nach GröBeneinstellung.

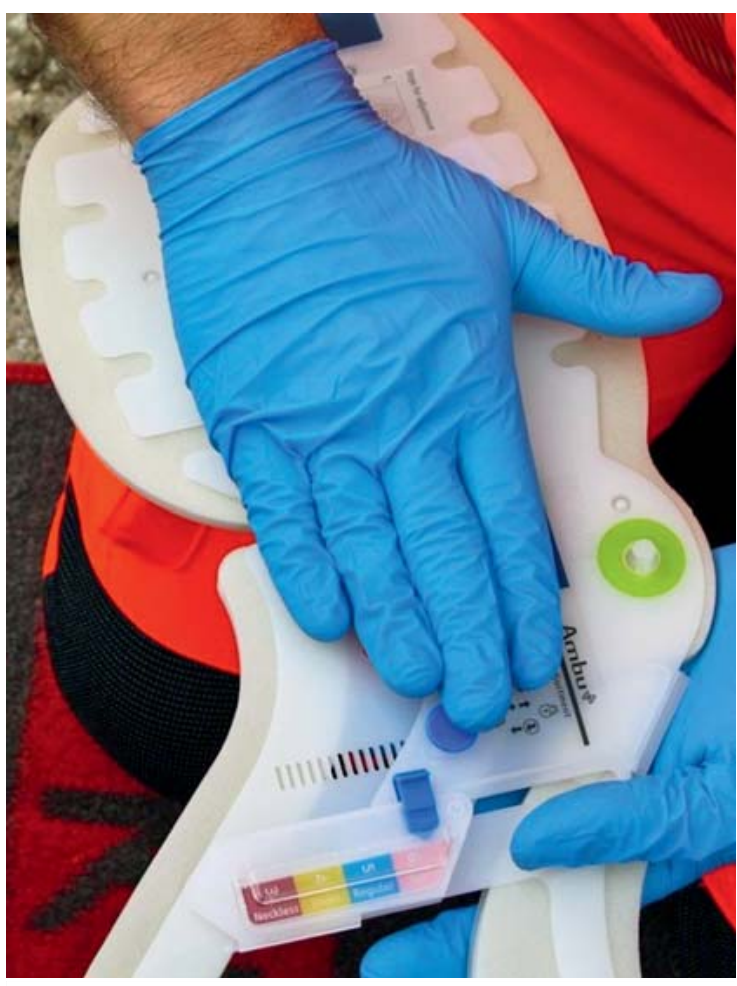

- Abb. 4 Größeneinstellung der Zervikalstütze anhand der ausgemessenen Halslänge; ggf. weitere Vorbereitung nach Herstellerangaben (z. B. Verriegelung).

\section{Schritt 3 Anlage der Zervikalstütze}

Die Zervikalstütze wird durch einen 2. Helfer am Patienten unter Fortführung der MILS positioniert, anmodelliert und straff mittels Klettband verschlossen ( $\bullet$ Abb. 5). Es muss bedacht werden, dass die alleinige Anlage einer Zervikalstütze keine ausreichende Stabilisierung der HWS ist und stets mit einer Ganzkörperimmobilisation kombiniert werden muss!

\section{Immobilisation des Patienten auf der Vakuummatratze}

Schritt 1 Positionierung des Patienten auf der Schaufeltrage

Zuerst wird die Länge der Schaufeltrage an die Körpergröße des Patienten angepasst ( $\vee$ Abb. $\mathbf{6 a}$ ), danach wird die Verriegelung zur Trennung der beiden Schaufeln gelöst ( Abb. 6b).

Nun kann das „Aufschaufeln“ des Patienten durch geringe, achsengerechte Drehung unter kontinuierlicher MILS erfolgen. Der Patient wird auf das Kommando des Kopfhelfers durch die anderen Helfer „en bloc“ etwas seitlich angehoben. Nun kann, wenn noch nicht erfolgt, der Rücken untersucht werden. Die beiden seitlich positionierten Helfer fassen den Patienten an Schulter, Becken und Oberschenkel unter Kreuzung ihrer Arme auf Beckenhöhe ( $\triangleright$ Abb. 7). So werden die Schaufeln von beiden Sei- 

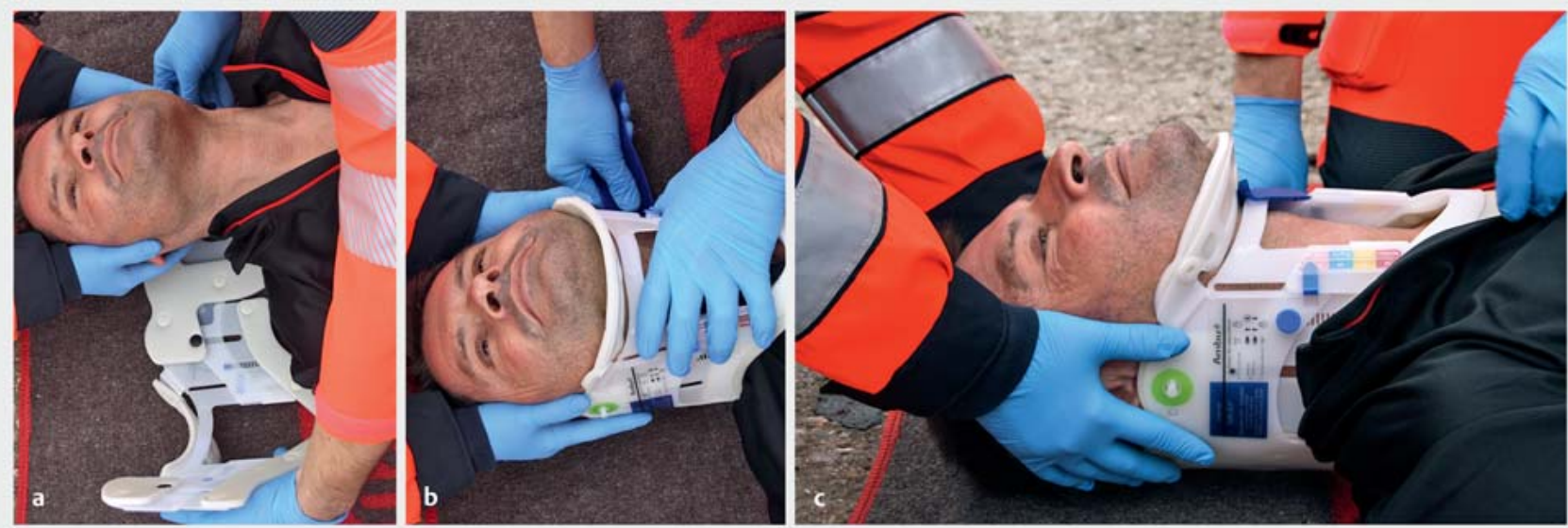

- Abb. 5 Anbringen der Zervikalstütze. a Positionierung der Zervikalstütze. b Anmodellieren und straffer Verschluss der Zervikalstütze mittels Klettband. c Patient mit fertig angelegter Zervikalstütze unter Fortführung der MILS.
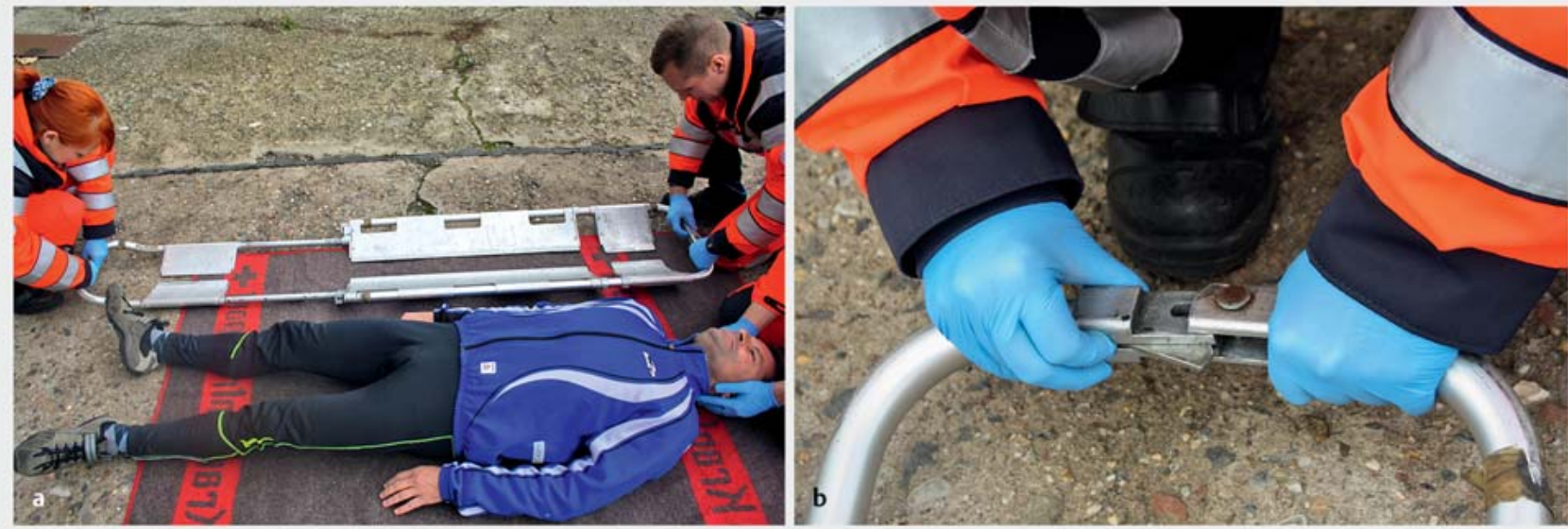

- Abb. 6 Benutzung der Schaufeltrage. a Anpassen der Länge der Schaufeltrage an die Körpergröße des Patienten. b Öffnen der Verriegelung zur Trennung der beiden Schaufeln.

ten eingebracht und nachfolgend die Verriegelung geschlossen. Mittels Gurtsystem wird der Patient nun auf der Schaufeltrage sicher fixiert ( $\bullet$ Abb. 8).

Schritt 2 Vorbereitung der Vakuummatratze und Umlagerung des Patienten

Die Vakuummatratze wird durch gleichmäßige Verteilung des Granulats, Auflage eines Umbetttuchs und leichtes Ansaugen vorbereitet. Nun wird der Patient mit der Schaufeltrage auf der Vakuummatratze gelagert. Im Anschluss werden die Gurte entfernt und beide Schaufelhälften, nach Entriegelung der Schaufeltrage, vorsichtig seitlich herausgenommen ( $\bullet$ Abb. 9).

Schritt 3 Immobilisation des Patienten auf der Vakuummatratze

Das Anmodellieren der Vakuummatratze erfolgt durch mehrere Helfer unter kontinuierlichem Absaugen der

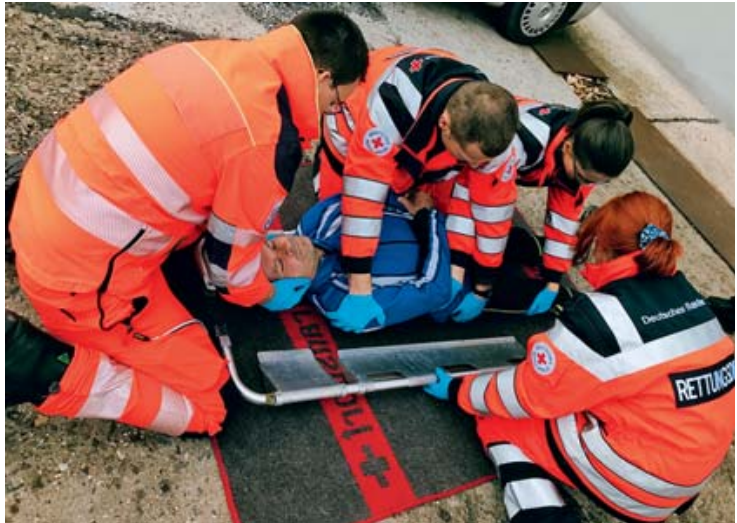

- Abb. 7 „Aufschaufeln“ des Patienten durch geringe, achsengerechte Drehung unter kontinuierlicher MILS. Die beiden seitlich positionierten Helfer fassen den Patienten an Schulter, Becken und Oberschenkel unter Kreuzung ihrer Arme auf Beckenhöhe. Einbringen der Schaufeln von beiden Seiten und nachfolgendes Schließen der Verriegelung. 


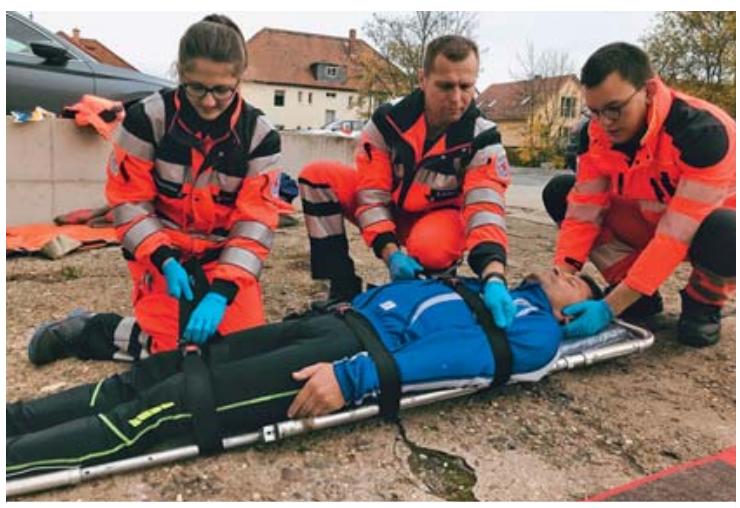

- Abb. 8 Sichere Fixierung des Patienten auf der Schaufeltrage mittels Gurtsystem.

Luft. Anschließend wird der Patienten mittels Gurtsystem gesichert ( $\triangleright$ Abb. 10). Ist eine sichere Fixierung des Kopfes mit dem Kopfteil der Vakuummatratze möglich, so kann nun die MILS aufgehoben bzw. auf die zusätzliche Anwendung einer Zervikalstütze verzichtet werden. - Abb. 11 zeigt den komplett immobilisierten Patienten nach Anbringen aller Sicherungsgurte der Vakuummatratze und der Fahrtrage.

\section{Immobilisation des Patienten auf dem Spineboard}

Schritt 1 Vorbereitung des Patienten zum Log-Roll-Manöver

Unter kontinuierlicher MILS fassen die beiden Seithelfer den Patienten an Schulter, Becken und Oberschenkel unter Kreuzung ihrer Arme auf Beckenhöhe. Das Spineboard wird parallel zum Patienten mit der Unterkante auf Höhe

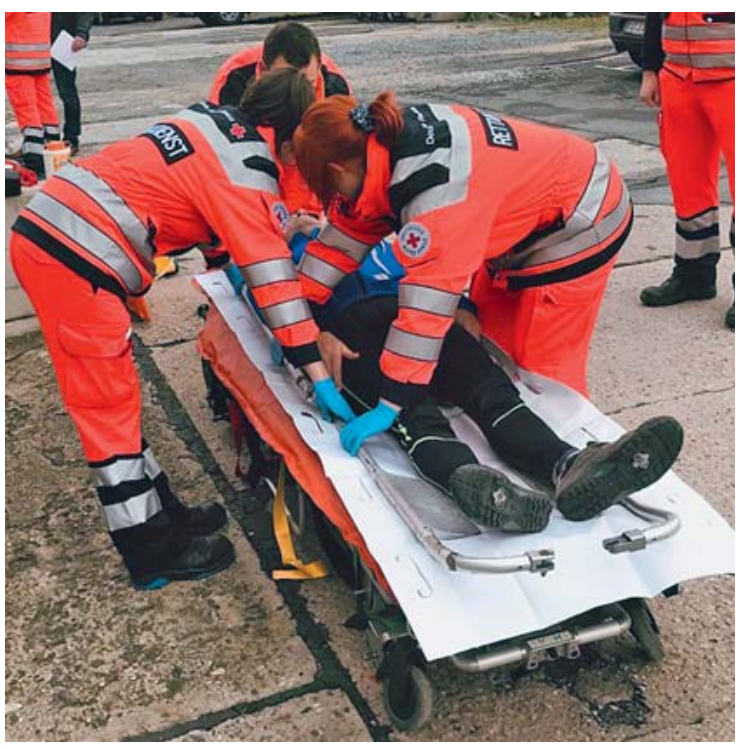

- Abb. 9 Nach Umlagerung des Patienten auf die bereits vorbereitete Vakuummatratze werden die Gurte entfernt, die Schaufeltrage entriegelt und beide Schaufelhälften vorsichtig seitlich herausgenommen.

von dessen Kniekehlen ausgerichtet ( $\triangleright$ Abb. 12). Der Patient wird auf das Kommando des Kopfhelfers durch die anderen Helfer „en bloc“ etwas seitlich angehoben. Nun kann, wenn noch nicht erfolgt, der Rücken untersucht werden.

\section{Schritt 2 Achsengerechtes Drehen des Patienten}

Das Spineboard wird an die Patientenrückseite gebracht und fixiert, wobei der Patient weiter kontinuierlich an Schulter und Becken stabilisiert wird ( $\bullet$ Abb. 13). Der Pa-
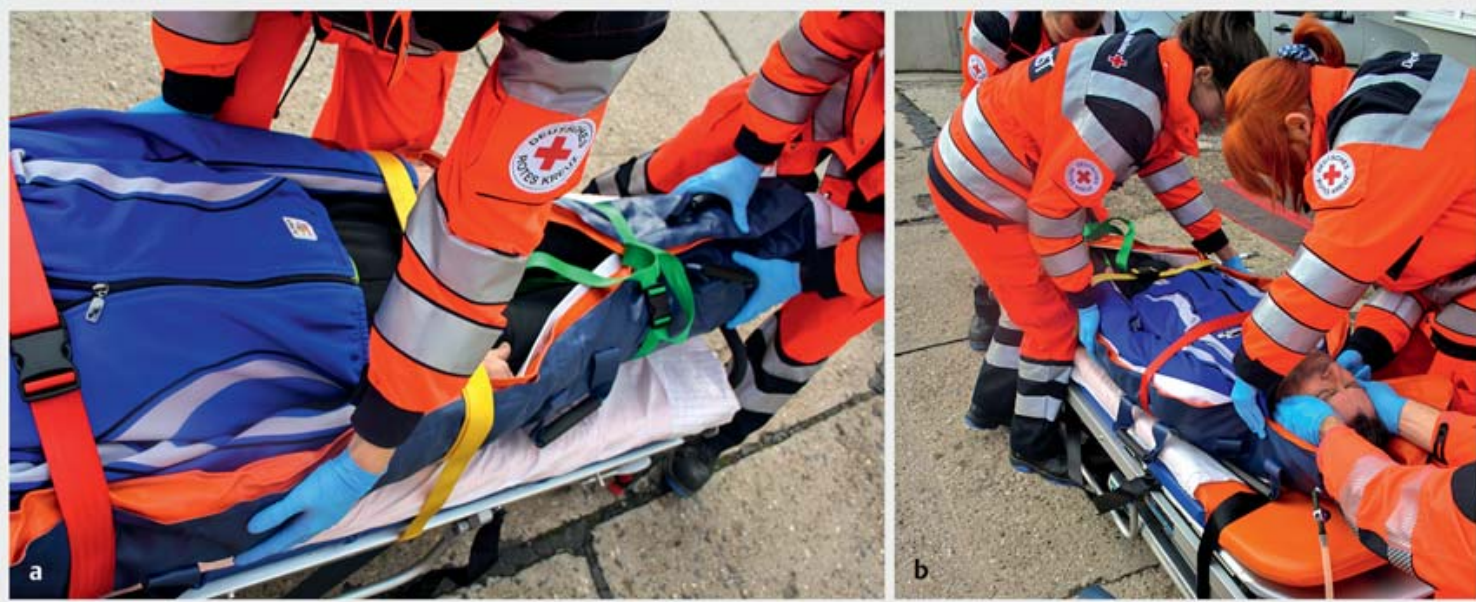

- Abb. 10 Anmodellieren der Vakuummatratze durch mehrere Helfer unter kontinuierlichem Absaugen der Luft. Anschließende Sicherung des Patienten mittels Gurtsystem. a Anmodellieren und Fixieren des unteren Teils der Vakuummatratze während des Absaugvorgangs. b Anmodellieren des Kopfteils der Vakuummatratze während des Absaugvorgangs unter kontinuierlicher MILS bis zur sicheren Fixierung. 


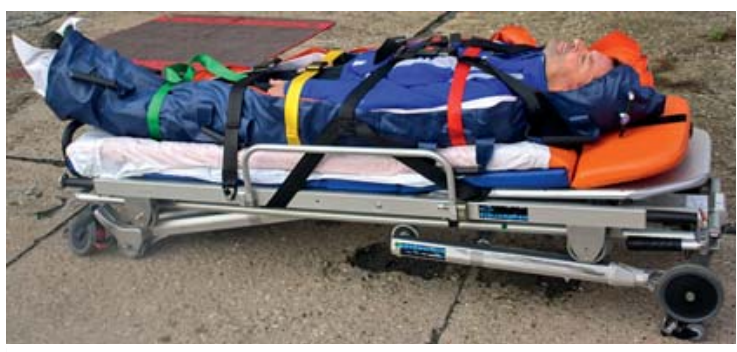

- Abb. 11 Komplett immobilisierter Patient in Vakuummatratze (Darstellung hier ohne angelegte Zervikalstütze) nach Anbringen aller Gurte (Vakuummatratze und Fahrtrage).

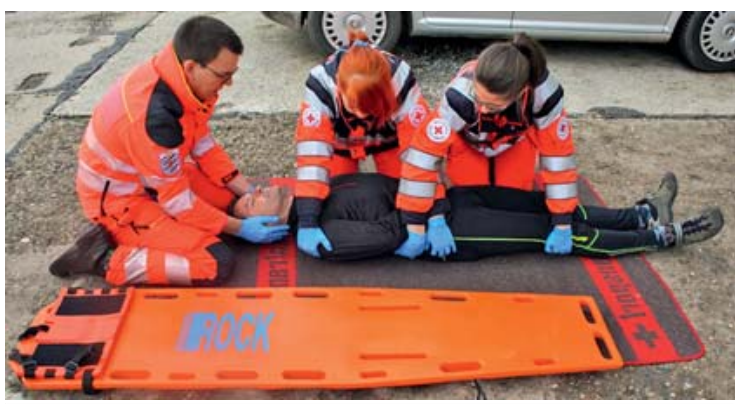

- Abb. 12 Vorbereitung des Patienten zum Log-Roll-Manöver: Die beiden seitlich positionierten Helfer fassen den Patienten an Schulter, Becken und Oberschenkel unter Kreuzung ihrer Arme auf Beckenhöhe. Der Kopfhelfer führt die MILS der HWS durch. Das Spineboard ist parallel zum Patienten (Unterkante auf Höhe der Kniekehlen des Patienten) ausgerichtet.

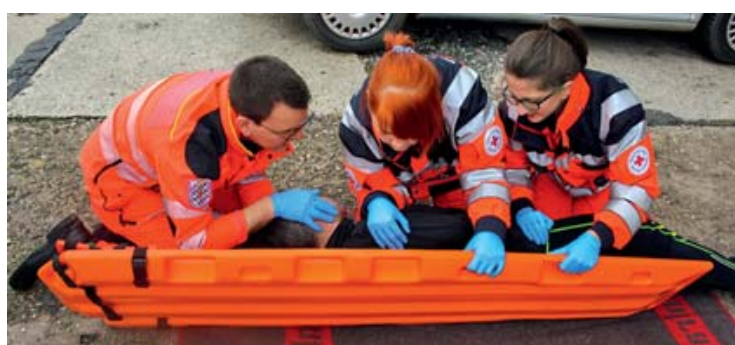

- Abb.13 Achsengerechtes Drehen des Patienten unter kontinuierlicher MILS: Heranbringen des Spineboards an die Patientenrückseite unter Fortführung der Stabilisierung an Schulter und Becken. Sodann Anpressen des Patienten mit den Unterarmen an das Spineboard und Zurückkippen in Rückenlage.

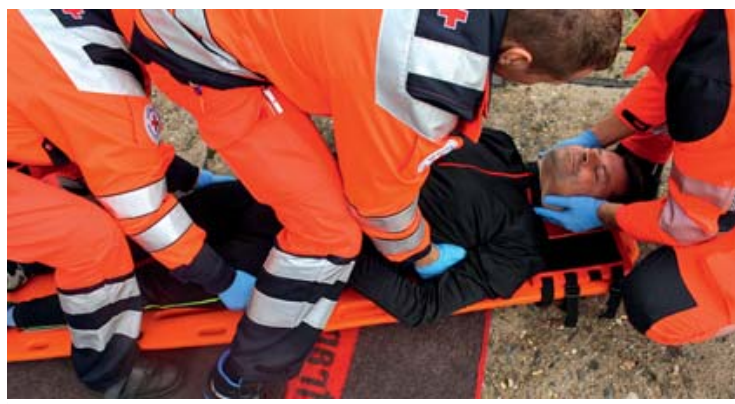

- Abb. 14 Korrektur der Patientenlage auf dem Spineboard: hierbei Fassen des Patienten unterhalb der Achseln am Thorax (Helfer 1) und am Becken (Helfer 2). Unter Beibehaltung der In-Line-Stabilisierung (Kopfhelfer) wird der Patient mittels longitudinalem Zug durch Helfer 1 unter Mitführung des Beckens durch Helfer 2 auf dem Spineboard gelagert.
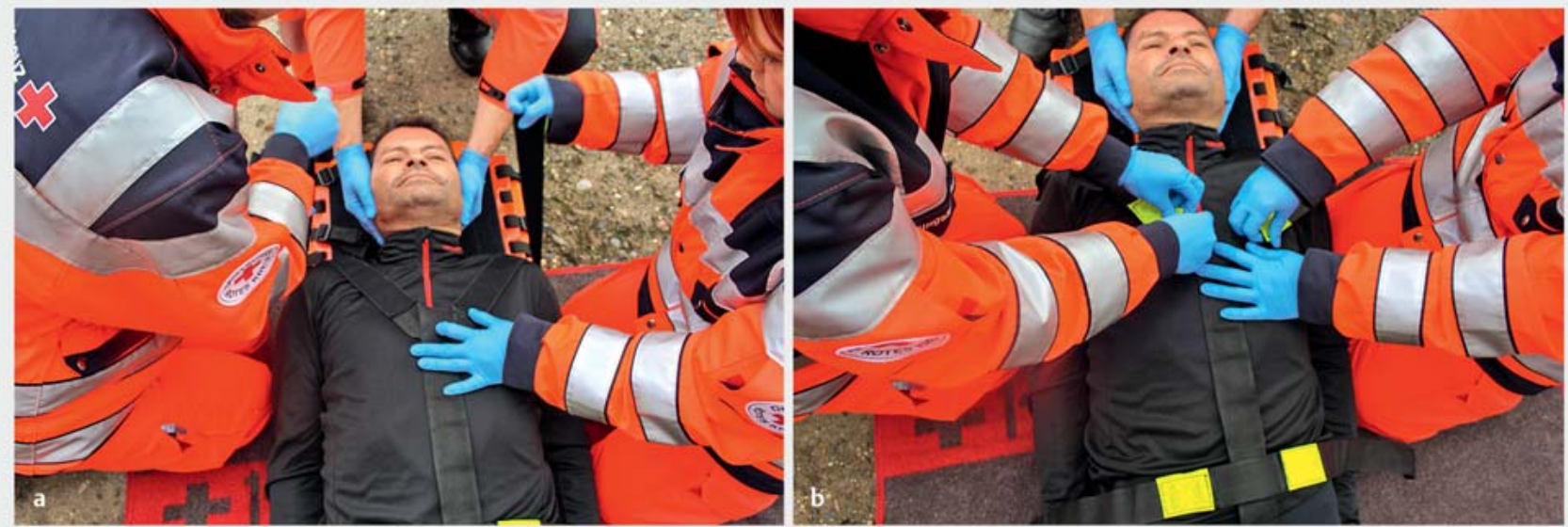

Abb. 15 Fixierung des Patienten auf dem Spineboard mittels Gurtspinne unter kontinuierlicher MILS. a Paralleles Anbringen der Gurte von beiden Seiten. b Schließen der Gurte beginnend von kranial nach kaudal. 


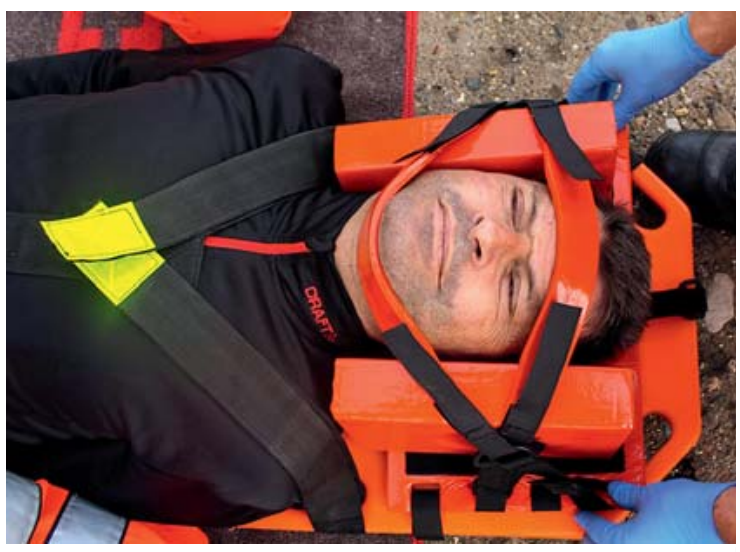

- Abb. 16 Die Head Blocks werden von beiden Seiten an den Kopf des Patienten herangeführt und am Spineboard fixiert. Komplette Immobilisierung mittels Stirn- und Kinngurt; die MILS kann jetzt aufgehoben werden (Darstellung hier ohne angelegte Zervikalstütze).

tient wird nun mit den Unterarmen an das Spineboard gepresst; danach vorsichtiges Zurückkippen von Patient und Board in Rückenlage.

\section{Schritt 3 Korrektur der Patientenlage auf dem Spineboard}

Zur Korrektur der Patientenlage auf dem Spineboard fassen die Helfer den Patienten unterhalb der Achseln am Thorax (Helfer 1) und am Becken (Helfer 2). Unter Beibehaltung der In-Line-Stabilisierung durch den Kopfhelfer wird der Patient nun mittels longitudinalem Zug durch Helfer 1 unter Mitführung des Beckens durch Helfer 2 auf dem Spineboard gelagert ( $\bullet$ Abb. 14).

\section{Schritt 4 Fixierung des Patienten auf dem Spineboard}

Der Patient kann nun auf dem Spineboard mittels Gurtspinne fixiert werden: paralleles Schließen der einzelnen Gurte von beiden Seiten - beginnend von kranial nach kaudal (॰ Abb. 15).

Als letzter Schritt werden die Head Blocks von beiden Seiten an den Kopf des Patienten herangeführt und am Spineboard fixiert. Mittels Stirn- und Kinngurt ist der Patient nun komplett immobilisiert, und die MILS kann aufgehoben werden bzw. auf die zusätzliche Anwendung einer Zervikalstütze kann verzichtet werden ( $\bullet$ Abb. 16).

\section{Interessenkonflikt}

Die Autoren geben an, dass keine Interessenkonflikte vorliegen.

\section{KERNAUSSAGEN}

- An eine Verletzung der Wirbelsäule ist bei jedem Traumapatienten zu denken.

- Bei gegebener Indikation zur Wirbelsäulenimmobilisation muss diese auch konsequent umgesetzt werden.

- Die Schaufeltrage und das Spineboard sind wichtige Devices zur wirbelsäulenschonenden Rettung.

- Zur weiterführenden Immobilisation ist die Vakuummatratze am besten geeignet.

\section{Autorinnen/Autoren}

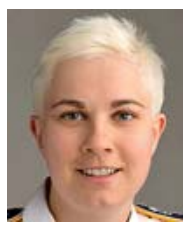

\section{Anne Weißleder}

Oberstabsarzt; Studium der Humanmedizin an der Friedrich-Schiller-Universität Jena. Assistenzärztin der Klinik für Anästhesiologie, Intensivmedizin, Notfallmedizin und Schmerztherapie am Bundeswehrkrankenhaus Ulm. Aktive Notärztin im RD-Bereich Saalfeld-Rudolstadt. Auslandseinsatz in Mali.

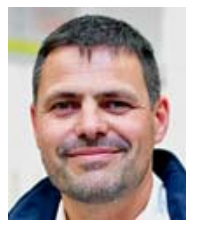

\section{Björn Hossfeld}

Dr. med., Oberfeldarzt; Studium der Humanmedizin an der Universität Würzburg. Facharzt für Anästhesiologie. Oberarzt an der Klinik für Anästhesiologie, Intensivmedizin, Notfallmedizin und Schmerztherapie am Bundeswehrkrankenhaus Ulm. Notarzt auf dem RTH Christoph 22. Mehrfache Teilnahme an Auslandseinsätzen der Bundeswehr in Afghanistan, Kosovo, Kongo und Niger.

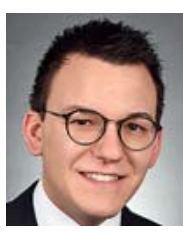

\section{Dominik Treffer}

Dr. med., Oberstabsarzt; Studium der Humanmedizin an der Universität Regensburg. Assistenzarzt an der Klinik für Anästhesiologie, Intensivmedizin, Notfallmedizin und Schmerztherapie am Bundeswehrkrankenhaus Ulm. Aktiver Notarzt und Kreisbereitschaftsarzt des BRK KV Eichstätt. Auslandseinsatz in Mali.

Hinweis

Dieser Beitrag ist eine aktualisierte Version des Artikels: Weißleder A, Helm M, Hossfeld B, Treffer D. Immobilisationstechniken der Wirbelsäule - Schritt für Schritt. Notfallmedizin up2date 2018; 13: 237-246

\section{Korrespondenzadresse}

\section{Anne Weißleder}

Oberstabsarzt

Klinik für Anästhesiologie, Intensivmedizin,

Notfallmedizin \& Schmerztherapie

Bundeswehrkrankenhaus Ulm

Oberer Eselsberg 40

89081 Ulm

anneweissleder@bundeswehr.org 


\section{Literatur}

[1] Schinkel C, Frangen TM, Kmetic A et al. Wirbelsäulenfrakturen bei Mehrfachverletzten: Eine Analyse des DGU-Traumaregisters. Unfallchirurg 2007; 110: 946-952. doi:10.1007/s00113007-1351-2

[2] Sektion NIS der Deutschen Gesellschaft für Unfallchirurgie (DGU); Akademie der Unfallchirurgie (AUC). TraumaRegister DGU ${ }^{\circledR}$ Jahresbericht 2014. Im Internet: http://www.dgu-online.de/fileadmin/published_content/5.Qualitaet_und_Sicherheit/PDF/2014_TR_DGU_Jahresbericht.pdf; Stand: 09.09.2018

[3] Stephan K, Huber S, Häberle S et al. Spinal cord injury - incidence, prognosis, and outcome: An analysis of the TraumaRegister DGU. Spine J 2015; 15: 1994-2001. doi:10.1016/j. spinee.2015.04.041

[4] Kwan I, Bunn F, Roberts I. Spinal immobilisation for trauma patients. Cochrane Database Syst Rev 2001; (2): CD002803. doi:10.1002/14651858.CD002803

[5] Sundstrøm T, Asbjørnsen H, Habiba S et al. Prehospital use of cervical collars in trauma patients: A critical review. J Neurotrauma 2014; 31: 531-540. doi:10.1089/ neu.2013.3094

[6] Kreinest M, Gliwitzky B, Schüler S et al. Development of a new Emergency Medicine Spinal Immobilization Protocol for trauma patients and a test of applicability by German emergency care providers. Scand J Trauma Resusc Emerg Med 2016; 24: 71. doi:10.1186/s13049-016-0267-7

[7] Arbeitsgemeinschaft der Wissenschaftlich Medizinischen Fachgesellschaften AWMF. S3-Leitlinie Polytrauma/Schwerverletzten-Behandlung. Im Internet: www.awmf.org/leitlinien/ detail/ll/012-019.html; Stand: 13.08.2018

[8] NAEMT, Hrsg. Präklinisches Traumamanagement: Prehospital Trauma Life Support (PHTLS), Deutsche Bearbeitung durch PHTLS Deutschland und Schweiz. 3. Aufl. München: Urban \& Fischer in Elsevier; 2016

[9] Hofmeister M, Bühren V. Therapiekonzept für Verletzungen der unteren HWS. Orthopäde 1999; 28: 401-413. doi:10.1007/PL00003624

[10] Schünke M, Schulte E, Schumacher U et al. Prometheus Allgemeine Anatomie und Bewegungssystem. 4. Aufl. Stuttgart: Thieme; 2014

[11] Adams HA, Baumann G, Gänsslen A et al. Die Definitionen der Schockformen. Intensivmed Notfallmed 2001; 38: 541-553. doi: $10.1007 / s 003900170030$

[12] Inaba K, DuBose J], Barmparas G et al. Clinical examination is insufficient to rule out thoracolumbar spine injuries. J Trauma 2011; 70: 174-179. doi:10.1097/TA.0b013e3181d3cc6e

[13] Singh Tveit M, Singh E, Olaussen A et al. What is the purpose of log roll examination in the unconscious adult trauma patient during trauma reception? Emerg Med J 2016; 33: 632-635. doi:10.1136/emermed-2015-205450

[14] Docimo S, Diggs L, Crankshaw L et al. No evidence supporting the routine use of digital rectal examinations in trauma patients. Indian J Surg 2015; 77: 265-269. doi:10.1007/s12262015-1283-y

[15] Hoffman JR, Mower WR, Wolfson AB et al. Validity of a set of clinical criteria to rule out injury to the cervical spine in patients with blunt trauma. National Emergency X-Radiography Utilization Study Group. N Engl J Med 2000; 343: 94-99. doi:10.1056/NEJM200007133430203

[16] Vaillancourt C, Stiell IG, Beaudoin T et al. The out-of-hospital validation of the Canadian C-Spine Rule by paramed- ics. Ann Emerg Med 2009; 54: 663-671.e1. doi:10.1016/j. annemergmed.2009.03.008

[17] Luscombe MD, Williams JL. Comparison of a long spinal board and vacuum mattress for spinal immobilisation. Emerg Med J 2003; 20: 476-478. doi:10.1136/emj.20.5.476

Bibliografie

DOI https://doi.org/10.1055/a-0797-5408

Notarzt 2018; 34: 326-334

(c) Georg Thieme Verlag KG Stuttgart · New York ISSN 0177-2309

\section{ZUSAMMENFASSUNG}

Verletzungen von Wirbelsäule und Rückenmark treten gehäuft bei polytraumatisierten Patienten auf. Da die Möglichkeiten der Diagnostik prähospital deutlich eingeschränkt sind, müssen derartige Verletzungsmuster durch den Notfallmediziner antizipiert werden. Dieser Artikel stellt wesentliche Befunde im Kontext der Wirbelsäulenverletzung dar und nennt Indikationen und Limitationen sowie etablierte Techniken zur prähospitalen Immobilisation der Wirbelsäule.

Wirbelsäule, Immobilisation, Polytrauma, Notfallmedizin

ABSTRACT

\section{Indications and Essential Tech- niques to Immobilize the Spine in a Prehospital Setting}

Trauma of the spine and medulla occur more frequently in severely injured patients. Due to very limited prehospital prospects of diagnostic, the emergency physician has to anticipate this injury patterns. This article presents substantial findings in the context of spinal injury and states indications, limitations as well as established techniques for a prehospital immobilization of the spine.

spine, immobilisation, polytrauma, emergency medicine 